\title{
Análisis bayesiano de factores de riesgo de accidente en trabajos de movimientos de tierras
}

\section{Bayesian analysis of risk associated with workplace accidents in earthmoving operations}

J. F. García ${ }^{(*)}$, J. E. Martín ${ }^{(*)}$, S. Gerassis ${ }^{(* *)}, \underline{\text { A. Saavedra }}^{(* * *)}$, J. Taboada García ${ }^{(* * * *)}$

\section{RESUMEN}

En este trabajo se analizan características de distintas obras en las que se ejecutaban trabajos de movimiento de tierras y tuvo lugar un accidente. Aplicando redes bayesianas se identifican los factores de mayor potencial predictivo de las situaciones de riesgo analizadas. Posteriormente se realizan estudios de inferencia para analizar la interrelación entre los distintos factores. Con todo esto se demuestra que las redes bayesianas pueden ser herramientas muy potentes en la descripción general de contextos de obra, y de gran capacidad predictiva dentro de la planificación de obras desde la perspectiva seguridad-producción.

Palabras clave: obra civil; movimiento de tierras; gestión de la seguridad; evaluación de riesgos; redes bayesianas; minería de datos.

\section{ABSTRACT}

This paper analyses the characteristics of earthmoving operations involving a workplace accident. Bayesian networks were used to identify the factors that best predicted potential risk situations. Inference studies were then conducted to analyse the interplay between different risk factors. We demonstrate the potential of Bayesian networks to describe workplace contexts and predict risk situations from a safety and production planning perspective.

Keywords: civil works; earthmoving; safety management; risk assessment; Bayesian networks; data mining.

(*) CIPP Internacional, S. L., Gijón, Asturias (España).

${ }^{(* *)}$ Departamento de Ingeniería de los Recursos Naturales y Medio Ambiente, Universidad de Vigo, Vigo, Pontevedra (España).

${ }_{(* * *)}$ Departamento de Estadística e Investigación Operativa, Universidad de Vigo, Vigo, Pontevedra (España).

(****) PhD student, Department of Natural Resources and Environmental Engineering, Universidad de Vigo, Vigo, Pontevedra (España).

Persona de contacto/Corresponding author: saavedra@uvigo.es (A. Saavedra)

ORCID: http://orcid.org/oooo-0002-6033-055X (J. F. García); http://orcid.org/oooo-0003-0157-2644 (J. E. Martín); http://orcid.org/oooo-0oo2-9249-6407 (S. Gerassis); http://orcid.org/oooo-0002-4845-2271 (A. Saavedra); http://orcid.org/oooo-0002-0298-8871 (J. Taboada García)

Cómo citar este artículo/Citation: García, J. F., Martín, J. E., Gerassis, S., Saavedra, A., Taboada García, J. (2017). Análisis bayesiano de factores de riesgo de accidente en trabajos de movimientos de tierras. Informes de la Construcción, 69(546): e192, doi: http://dx.doi. org/10.3989/ic.15.154.

Copyright: (C) 2017 CSIC. Licencia / License: Salvo indicación contraria, todos los contenidos de la edición electrónica de Informes de la Construcción se distribuyen bajo una licencia de uso y distribución Creative Commons Attribution License (CC BY) Spain 3.o. 


\section{INTRODUCCIÓN}

La seguridad en el trabajo es un elemento clave para el desarrollo óptimo de las actividades laborales. El sector de la construcción es, desde el punto de vista de la siniestralidad laboral, uno de los más problemáticos. Sus tasas de siniestralidad están entre las más elevadas, y resultan aún más llamativas porque sus incrementos, en fases de crecimiento de los países, son trágicos (1).

Son muchos los factores que determinan el contexto en que se acomete una obra. La búsqueda de modelos que cuantifiquen numéricamente el riesgo de accidente durante la fase de planificación de la obra es una tarea de suma importancia. Algunos autores han investigado sobre metodologías que permitan identificar factores de riesgo en trabajos de construcción (2) (3) e incluso clasificarlos y ponderarlos en función de su peligrosidad (4). Otros autores, como (5), han desarrollado sistemas de información para la prevención de accidentes orientados a la identificación de factores de riesgo y la evaluación del riesgo.

En las obras de construcción, y en el movimiento de tierras en particular, es dificultoso efectuar las evaluaciones de riesgo de cada puesto de trabajo debido a la movilidad de los mismos, realización de multitareas y los continuos entornos cambiantes. Por esta razón, el poder predecir en cada uno de los contextos en que se va a acometer una obra el riesgo potencial de accidente sería una capacidad muy potente en el campo de la siniestralidad laboral.

Lo sería aún más si los técnicos encargados de evaluar estos entornos fueran capaces de cuantificar como variaría la probabilidad de que tuviera lugar un potencial accidente en función de las acciones que se adoptasen en materia preventiva, ya que así se podrían planificar las tareas del movimiento de tierras desde la perspectiva del binomio seguridad-producción.

Con el término movimiento de tierras consideramos el conjunto de operaciones que se realizan con los terrenos naturales, para dotarlos de unas determinadas características geométricas y mecánicas, que vendrán determinadas por su posterior aplicación. En la construcción de grandes obras lineales (carreteras, autopistas, líneas ferroviarias, etc.), de áreas industriales, de urbanizaciones, etc., el movimiento de tierras constituye un capítulo cuyo peso presupuestario es variable en función de las características de la obra, pudiendo llegar incluso, en alguna ocasión excepcional, a suponer el de más relevancia en la construcción de algunas de ellas.

La metodología utilizada para el análisis de los distintos contextos es la red bayesiana. Las redes bayesianas son modelos gráficos probabilísticos utilizados en la toma de decisiones (6). Se caracterizan por su simplicidad interpretativa ya que las relaciones entre los factores que definen el contexto se representan mediante redes. Son herramientas de predicción muy potentes puesto que permiten estudiar contextos nuevos y sus posibles consecuencias. Además, permite llevar a cabo estudios de sensibilidad cuantificando el impacto que pequeñas variaciones en alguno de los factores pueden provocar en el resto de los factores. Las redes bayesianas han sido empleadas en estudios tan diversos como la evaluación de la calidad de techos de pizarra (7), el análisis de accidentes de trabajo causados por caídas desde altura (8), la predicción de desastres naturales (9), predicción de accidentes en lugares de trabajo (10) o la evaluación del riesgo de accidente en proyectos de construcciones de acero (11).

Desde hace años distintos grupos de investigación han utilizado las redes bayesianas como una herramienta de gran utilidad en el estudio de los accidentes laborales. En primer lugar ofrecen al experto una visión muy ajustada a la realidad del alcance de las causas que son desencadenantes de los accidentes que se deseen evaluar en función de cada uno de los factores implicados. En segundo lugar facilitan la definición de la estructura de las relaciones entre factores causales que resulta de gran utilidad para identificar el impacto que tienen determinadas situaciones sobre los distintos tipos de accidentes que pueden acontecer en las empresas.

En este artículo se presenta el potencial que tienen las redes bayesianas en la evaluación de contextos generales de obra, como herramienta de apoyo para la elaboración de estudios y planes de seguridad en obras de construcción. Concretamente se estudian las características de distintas obras en las que se ejecutaban trabajos de movimiento de tierras. Para ello se entrenan redes, empleando para ello una base de datos creada con los accidentes sobrevenidos durante la ejecución de los trabajos de movimiento de tierras.

\section{MATERIALES Y MÉTODOS}

\subsection{Conceptos de teoría bayesiana}

Las redes bayesianas modelizan fenómenos aleatorios mediante la representación de un conjunto de factores y las relaciones de dependencia entre ellas. Estas relaciones se representan a través de modelos gráficos, habitualmente grafos acíclicos dirigidos.

Una red bayesiana se define como una tripleta $(X, G, P)$ en donde $X=\left(X_{1}, X_{2}, \ldots, X_{n}\right)$ es el conjunto de factores del dominio. $G$ es un grafo acíclico dirigido cuyos nodos están etiquetados con los elementos de $X$ y los arcos dirigidos indican relación de influencia y, en algunos casos, relación causal. $P$ es una distribución conjunta de probabilidad sobre $X$.

Aplicando resultados básicos de cálculo de probabilidades es fácil comprobar que la distribución conjunta $P$ puede expresarse como producto de probabilidades condicionadas de forma que:

$P(X)=P\left(X_{1}, X_{2}, \ldots, X_{n}\right)=P\left(X_{1}\right) \prod_{i=2}^{n} P\left(X_{i} \mid X_{1}, \ldots, X_{i-1}\right)$

Además, cada factor tiene asociada una tabla de probabilidades condicionales que definen la probabilidad de cada estado en los que puede estar ese factor: $P\left(X_{i}=x \mid X_{1}, \ldots, X_{i-1}, X_{i+1}, \ldots\right.$, $X_{n}$ ). Es fácil intuir que si el número de factores del dominio es muy elevado, la parte cuantitativa de la red puede llegar a hacerse inasumible en términos de tiempo de computación.

Con el fin de simplificar este planteamiento, las redes Bayesianas asumen, mediante el teorema de descomposición, que un nodo depende solamente de sus padres, entendiendo por nodo padre aquél del cual es descendiente mediante un arco del grafo $G$. Dicho de otra forma, el nodo padre es el nodo causa y su descendiente es nodo efecto. Así, el teorema de descomposición permite calcular la probabilidad conjunta $P$ 
bajo la hipótesis de que cada nodo es, dados sus padres, independiente de sus no-descendientes. De esta manera, dada una red bayesiana $(X, G, P)$ con factores $X=\left(X_{1}, X_{2}, \ldots, X_{n}\right)$, entonces la distribución de probabilidad conjunta $P$ de estos factores puede descomponerse de la forma:

$$
P(X)=P\left(X_{1}, X_{2}, \ldots, X_{n}\right)=\prod_{i=1}^{n} P\left(X_{i} \mid X_{j(i)}\right)
$$

Donde $X_{j(i)}$ denota el conjunto de factores padre de $X_{i}$ según el grafo $G$. Como consecuencia, para especificar una red bayesiana es necesario dar, para cada factor, una distribución de probabilidad condicionada dados sus padres. Si el factor es nodo raíz del grafo $G$, la distribución será la distribución marginal, ya que no tiene padres. Por tanto, utilizando el teorema de descomposición, cada estado de un factor puede ser calculado multiplicando un número reducido de valores en las tablas de probabilidad.

\subsubsection{Fase de aprendizaje de la red bayesiana}

La determinación del grafo $G$ que relaciona los factores $X$ y la distribución de probabilidad conjunta $P$ puede llevarse a cabo según tres procedimientos diferentes:

- A partir del conocimiento de expertos, que son los que determinan la estructura de la red, decidiendo la dirección de los arcos (relaciones causa-efecto). También deben determinar la estructura de dependencia entre los factores representados por los nodos así como sus probabilidades.

- Otro procedimiento sería mediante aprendizaje automático a partir de bases de datos. En la mayoría de los casos se realiza mediante la implementación de algoritmos específicos. El aprendizaje suele consistir en una primera fase de aprendizaje estructural, en la cual se deduce el grafo o topología de la red y una segunda fase de aprendizaje paramétrico en la cual se obtienen las probabilidades asociadas a cada nodo. Estas tablas de probabilidad se calculan de acuerdo a la estructura y la información suministrada en la base de datos. Cuando se tienen datos completos y suficientes para todos los factores en el modelo, es relativamente fácil obtener estas probabilidades, asumiendo que la estructura está dada. El método más común es el llamado estimador de máxima verosimilitud, bajo el cual se estiman las probabilidades en base a las frecuencias de los datos.

- Por último, también es posible la implementación de modelos mixtos, en los cuales se combinan el conocimiento experto con bases de datos en el aprendizaje de la red bayesiana.

\subsubsection{Tipos de inferencia de la red bayesiana}

Las redes bayesianas se pueden utilizar para realizar razonamientos en cualquier dirección. Así, pueden llevarse a cabo razonamiento predictivo, es decir desde nodos causa hasta nodos efectos siguiendo el sentido de los arcos que los conectan, o pueden realizarse razonamiento de diagnóstico, desde nodos efecto hasta nodos causa en sentido contrario a los arcos de la red. Otro tipo de inferencia posible es el razonamiento intercausal. En este caso se puede estudiar la relación entre distintos nodos causa que tienen en común un mismo nodo efecto.

En el presente trabajo, tanto la fase de aprendizaje como de inferencia de las redes bayesianas han sido llevadas a cabo con el software GeNIe \& SMILE (12) desarrollado por la Universidad de Pittsburgh.

\subsection{Recogida de datos y definición de factores}

La base de datos incluye 417 accidentes de trabajo comprendidos en un período de 6 años de investigación. Se incluyen accidentes pertenecientes a un grupo empresarial español del sector de la construcción especializado en el movimiento de tierras. La base de datos fue creada a partir de las empresas principales que forman el grupo, así como de las contratas que trabajan para el mismo.

Previo a la aplicación del modelo bayesiano, y como en el caso de cualquier otro sistema experto o herramienta estadística, es necesario identificar y definir los factores a partir de los datos disponibles, teniendo en cuenta una serie de hipótesis de trabajo de partida. Así, para la definición de factores se han seleccionado distintos parámetros que interesaba evaluar en base a los distintos problemas planteados. Para ello se dispone de los datos extraídos de dos fuentes: el parte de accidente y las entrevistas a pie de campo, o vía telefónica, de los jefes de obra y/o técnicos de prevención que en su día intervinieron en la investigación de los sucesos. De ambas se recoge información acerca de factores asociados al movimiento de tierras. Algunos son genéricos y pueden afectar a la ocurrencia de cualquier accidente y otros son concretos y atienden a la especificidad de la situación de obra analizada.

Los factores elegidos para evaluar el movimiento de tierras, así como sus posibles estados, son los siguientes:

- Fo1. Turnicidad:

S1.- No. Un único relevo de trabajo al día.

S2.- Sí. Dos o más relevos de trabajo al día.

- Fo2. Duración de cada relevo de trabajo:

S1.- Jornadas laborales de 8 horas o menos.

S2.- Jornadas laborales de duración superior a 8 horas.

En ambos casos, con independencia del número de turnos diarios.

- Fo3. Antigüedad de la maquinaria:

S1.- La totalidad de la maquinaria empleada en los trabajos de movimiento de tierras tiene una fecha de fabricación inferior a 10 años.

S2.- Existe maquinaria con fecha de fabricación superior a 10 años.

- Fo4. Mantenimiento de la maquinaria:

S1: Se caracteriza el mantenimiento como bueno cuando en el programa de trabajos está implantado un tiempo de dedicación al mantenimiento por parte de los operarios de la maquinaria y se comprueba el seguimiento de su cumplimiento, por parte de los responsables mecánicos, encargados y jefatura de obra.

S2: No existe tiempo destinado al mantenimiento en la organización del trabajo o no existe el referido seguimiento de su cumplimiento.

- Fo5. Estado de tajos y pistas de acarreo:

S1.- Se caracteriza el estado de tajos y pistas como bueno cuando la comprobación de los parámetros relacionados es favorable y se observa la disposición de los recursos adecuados para su mantenimiento, motoniveladoras, cubas de riego, etc.

S2.- Se evidencian carencias, en cuanto a que presentan elevadas pendientes, curvas sin visibilidad, mal peraltado, 
baches, blandones, materiales sueltos en zonas de paso de vehículos de acarreo o polvo.

- Fo6. Estabilidad de taludes de excavación:

S1.- Frentes de excavación donde no se hayan producido desprendimientos o se observen condiciones susceptibles de que se produzcan.

S2.- Frentes donde se hayan producido desprendimientos o se dan condiciones para que se produzcan.

- Fo7. Tipo de vehículos de acarreo:

S1.- En la obra se emplean dúmperes extraviales rígidos para el acarreo de tierras.

S2.- Se emplean dúmperes extraviales articulados.

S3.- Se emplean camiones viales, de 2, 3, 4 o 5 ejes.

- Fo8. Interferencia con otra maquinaria:

S1.- No. No existe interferencia con equipos de trabajo de otras operaciones.

S2.- Sí. Existe interferencia con otra maquinaria: de movimiento de tierras de otras empresas, grúas, cubas de hormigón, etc.

- Fo9. Control de aguas en pistas y tajos de carga y descarga: S1- Buen drenaje. No existe agua, ya sea porque no aparece o porque el drenaje es bueno.

S2- Presencia de agua.

- F10. Generación de polvo en pistas:

S1.- Ocasional. Presencia de polvo ocasional, derivada de una actuación eficaz de dichos equipos.

S2.- Frecuente. Presencia de polvo frecuente.

- F11. Subcontratación en el mismo tajo:

S1.- No. La propia empresa de movimiento de tierras ejecuta todas las unidades que tiene contratadas sin subcontratar.

S2.- Sí. La empresa de movimiento de tierras subcontrata operaciones, como, por ejemplo, la perforación y voladura, maquinaria, cubas de agua, estabilización de suelos, etc.

- F12. Señalización y balizamiento:

S1.- Correcta. Existe una señalización correcta y visible en la obra, indicando claramente por donde han de circular los peatones, las limitaciones de velocidad, zonas donde se realizan trabajos puntuales en las proximidades de las pistas de acarreo, etc.

S2.- Incorrecta. No existe señalización o no está correctamente colocada en la obra.

- F13. Orden y limpieza en la obra:

S1.- Buena. La obra presenta buen estado de orden y limpieza.

S2.- Con carencias. No se cumplen las condiciones anteriores.

- F14. Temperatura en la zona de obra:

S1.- Se alcanzan temperaturas superiores a los $30^{\circ} \mathrm{C}$ en la obra.

S2.- Las temperaturas no bajan de $\operatorname{los} \mathrm{O}^{\circ} \mathrm{C}$, ni superan los $30^{\circ} \mathrm{C}$.

$\mathrm{S}_{3}$ - Temperaturas en obra por debajo de $\operatorname{los} \mathrm{O}^{\circ} \mathrm{C}$.

- F15. Plazo de ejecución:

S1.- Obra en plazo. La obra se ha desarrollado en todo momento de acuerdo al Plan de Obra, con cumplimiento de los plazos de ejecución.

S2.- Obra con retraso. La obra, en algún momento de su ejecución, ha incurrido en retrasos en los plazos de cumplimiento del Plan de Obra.

- F16. Accidente realizando la siguiente operación:

S1.- El accidente se ha producido efectuando operaciones de arranque y carga. Maquinaria: retroexcavadora, pala cargadora.

S2.- El accidente se ha producido efectuando operaciones de acarreo, con dúmperes, lagartos, camiones, bañeras, etc.
S3.- El accidente se ha producido en operaciones de extendido y compactado de terraplenes, explanadas, tierra armada o extendidos en vertederos y acopios. Maquinaria: buldózer, compactador, motoniveladora, cubas de agua.

- F17. Hora del accidente:

S1.- Mañana. Comprende el período que va desde la incorporación matutina al puesto de trabajo hasta la hora del almuerzo.

S2.- Tarde. Comprende el período vespertino y nocturno. S3.- Horas extras. Período que sobrepase las 8 horas de trabajo, tanto en horario matutino, vespertino como nocturno.

- F18. Día del accidente:

S1.- El accidente tuvo lugar un lunes.

S2.- El accidente tuvo lugar un martes, miércoles o jueves.

S3.- El accidente tuvo lugar un viernes o sábado.

- F19. Tiempo del operario en la obra (antigüedad):

S1.- El operario objeto de accidente llevaba menos de 3 meses trabajando en la obra.

S2.- El operario accidentado llevaba trabajando en la obra entre 3 meses y 1 año.

S3.- El operario accidentado llevaba trabajando en la obra más de 1 año.

- F20. Formación:

S1.- El trabajador accidentado había sido formado antes de empezar su actividad en la obra.

S2.- El trabajador accidentado había sido formado una vez empezada su actividad en la obra.

S3.- El trabajador accidentado aún no había recibido formación cuando tuvo lugar el accidente.

- F21. Edad:

S1.- El trabajador accidentado tenía una edad comprendida entre los 18 y 30 años.

S2.- El trabajador accidentado tenía una edad comprendida entre los 30 y 55 años.

S3.- El trabajador accidentado tenía más de 55 años.

- F22. ¿Era su trabajo habitual?:

S1.- Sí. El trabajador accidentado se encontraba efectuando la tarea para la que había sido contratado.

S2.- No. El trabajador accidentado no se encontraba efectuando la tarea para la que había sido contratado.

- F23. Nacionalidad:

S1.- El trabajador accidentado era de nacionalidad española. S2.- El trabajador accidentado no era de nacionalidad española.

- F24. Tipo de contrato:

S1.- Indefinido. El trabajador accidentado tenía un contrato indefinido en el momento del accidente.

S2.- Temporal. El trabajador accidentado tenía un contrato temporal en el momento del accidente.

Además de los 24 factores anteriores, se utiliza en las redes un factor de predicción que tiene como estados los correspondientes tipos de accidentes acaecidos en las obras. Estos son:

- CMN: Caídas de personas al mismo y/o distinto nivel.

- CMD: Caídas de objetos desprendidos, por manipulación, desplome o derrumbamiento.

- PSO: Pisadas sobre objetos.

- COI: Choques contra objetos móviles y/o inmóviles.

- GOH: Golpes por objetos o herramientas.

- PFP: Proyección de fragmentos o partículas.

- AOM: Atrapamiento por o entre objetos y/o vuelco de máquinas. 
- SOB: Sobreesfuerzos.

- EXP: Explosiones.

- AGV: Atropellos o golpes con vehículos.

\section{RESULTADOS}

Se identificaron y definieron cada uno de los factores procedentes de las encuestas y/o partes de accidente de trabajo evaluados. Para la fase de aprendizaje de las redes bayesianas se utilizó un modelo mixto, combinando el conocimiento de un experto en prevención de riesgos laborales para el diseño de la red junto con bases de datos para el aprendizaje de las probabilidades de los nodos.

Una vez expuesto todo el proceso referente a metodología y estrategias seguidas en el desarrollo de la investigación, se muestran a continuación ejemplos de distintos contextos y/o entornos que verificarán el potencial de la herramienta creada.

\subsection{Análisis global de la unidad de obra «movimiento de tierras»}

En este estudio se realiza un planteamiento general de todos los factores que se han seleccionado para evaluar el entorno global de las obras de movimiento de tierras.

Los factores seleccionados para el movimiento de tierras han sido 24, según los criterios ya expuestos en la sección anterior. El nodo final de la red es, en este caso, el tipo de accidente.

Según lo mostrado en la Figura 1 para el colectivo afectado en la unidad de obra «movimiento de tierras» se deducen los siguientes resultados:
- El accidente más probable (25\% de los casos) es el derivado de las caídas al mismo y/o distinto nivel.

- La práctica totalidad de las empresas, $91 \%$, que efectuaban el movimiento de tierras trabajan a turnos.

- La subcontratación de trabajos en el mismo tajo era habitual superando los relevos de trabajo las 8 horas de duración.

- La antigüedad de las máquinas en obra (72\%) superan los 10 años, efectuándose el mantenimiento de éstas (82\%) con algún tipo de carencia.

- En cuanto al estado de los tajos y las pistas de acarreo cabe decir que en su mayor parte (92\%) presentan alguna carencia desde el punto de vista preventivo.

- Los taludes de excavación (76\%) presentaban problemas de estabilidad derivados del deslizamiento o corrimiento de tierras.

- La interferencia entre máquinas es el estado más frecuente en el movimiento de tierras (98\%), siendo el dúmper el vehículo de acarreo más utilizado (58\%).

- En los tajos de carga y descarga la presencia de agua era habitual (95\%), siendo la presencia de polvo frecuente en un $41 \%$ de los casos.

- En la mayor parte de las obras (58\%) la señalización y balizamiento cumplía los estándares que dicta la normativa correspondiente.

- El estado habitual en las obras, en lo que al orden y limpieza se refiere, era deficiente en la mayoría de las situaciones en las que aconteció un accidente (95\%).

- El rango de temperatura a la que se encontraban los trabajadores en obra era entre o y $30^{\circ} \mathrm{C}(79 \%)$.

- La práctica totalidad de los movimientos de tierras evaluados en obra (98\%) no se ejecutaron en el plazo previsto.

- La operación ejecutada en el momento que tenía lugar un accidente más habitual (36\%) era la de formación de terraplenes.

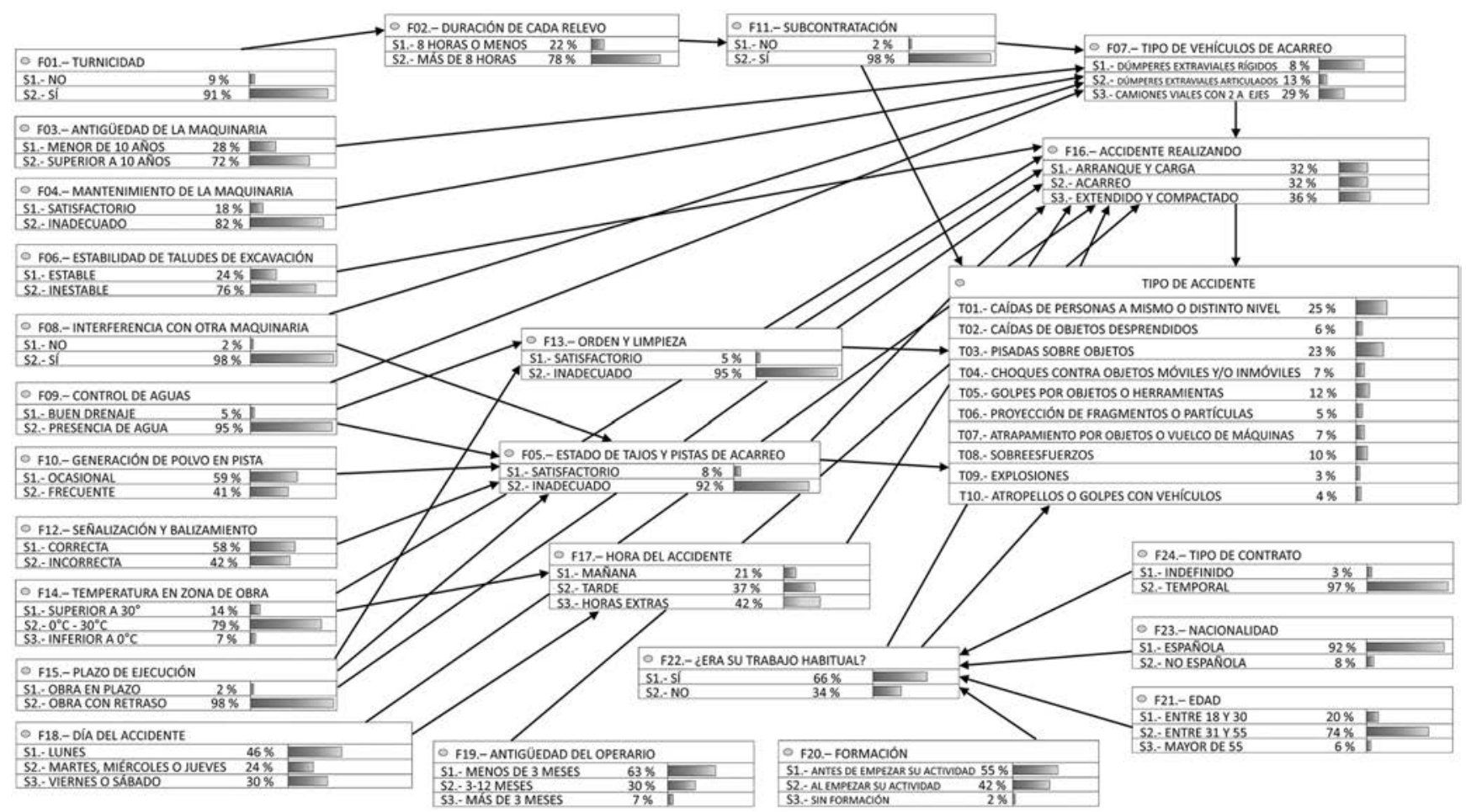

Figura 1. Red bayesiana obtenida en el análisis de la accidentalidad proporcionadas por los datos para los accidentes acaecidos en la unidad de obra «movimiento de tierras». 


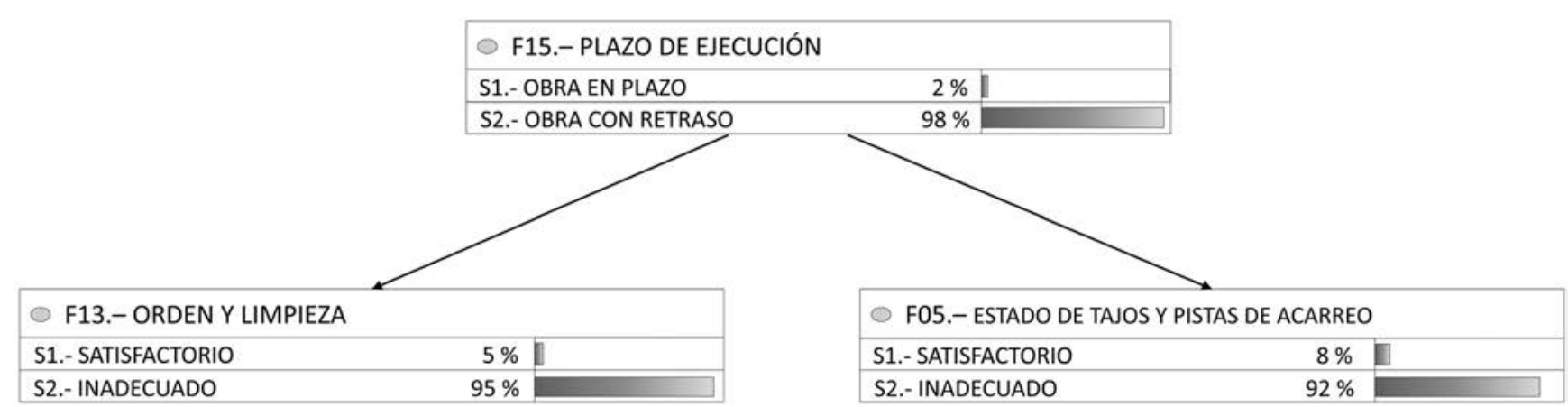

Figura 2. Relación intercausal entre los factores F15, F13 y Fo5 en el movimiento de tierras.

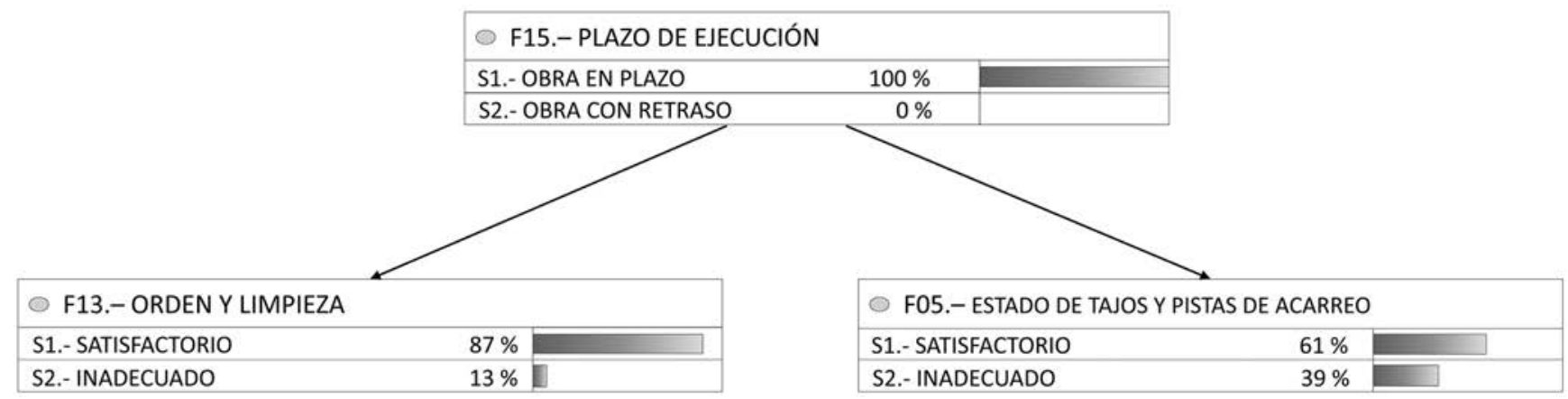

Figura 3. Relación intercausal entre los factores F15, F13 y Fo5 en el movimiento de tierras, bajo la hipótesis de que todas las obras se ejecutaron según los plazos contratados.

- El lunes es el día en que acaecieron más siniestros (46\%), siendo éstos, en su mayor parte (42\%), cuando se ejecutaban horas extras.

- El trabajador tipo que ejecuta los trabajos de movimientos de tierras llevaba más de un año en obra (63\%), es de nacionalidad española (92\%), fue formado antes de empezar los trabajos en obra ( $56 \%$ ) y tenía una edad comprendida entre los 30 y 55 años (o,74\%). En el momento del accidente efectuaba el trabajo para el que se había contratado (66\%), siendo su contrato, en el $97 \%$ de los casos, de tipo temporal.

Cabe además destacar otras conclusiones que se aprecian desde el punto de vista preventivo en este contexto global de las obras. Seleccionando en el nodo final cada uno de los tipos de accidente, y aplicando técnicas de razonamiento de diagnóstico, es posible realizar un análisis más detallado. Así:

- Un $34 \%$ de los trabajadores que tuvieron un accidente en este tipo de trabajos no estaban ejecutando el trabajo para el que habían sido contratados.

- Aunque la práctica totalidad de los trabajadores (97\%) han sido formados en materia preventiva, cabe destacar que un gran porcentaje ( $43 \%$ ) no ha sido formado según dicta la normativa de prevención de riesgos laborales.

- La mayor parte de los accidentes (76\%) se concentraron en los lunes y/o viernes.

- La señalización y/o balizamiento, en un $42 \%$ de los contextos de obra analizados, presentaba alguna deficiencia o ésta era inexistente.

- En el $41 \%$ de las situaciones de obra en las que tuvo lugar un accidente la presencia de polvo en pistas era notable.

- En cuanto a los accidentes acaecidos, el 50\% se concentraron en caídas de personas al mismo y/o distinto nivel y pisadas sobre objetos.

\subsection{Ejemplos de inferencia entre factores que afectan al binomio prevención-producción}

Otra de las bondades de las redes bayesianas es que, una vez han sido entrenadas, permiten realizar razonamiento intercausal entre factores significativos en un determinado tipo de accidentes y/o situaciones de obra. Éste es el caso del plazo de ejecución de las obras. Este factor puede tener una connotación importante desde un punto de vista económico ya que pueden existir penalizaciones por incumplimiento del contrato de no finalizarse la ejecución de los trabajos según los plazos pactados.

En los trabajos de movimiento de tierras objeto de este artículo (Figuras 1 y 2) es llamativo el hecho de que el $98 \%$ de éstos sufrían retrasos de ejecución. Igualmente se observa que en la mayor parte de las ocasiones (Figura 2), tanto las condiciones de orden y limpieza (95\%) como el estado de los tajos (92\%), presentaban algún tipo de carencia.

Estos dos factores tienen una importante incidencia en la correcta praxis de los trabajos influyendo en el plazo de ejecución de la obra. Para corroborar esta afirmación se realiza una inferencia bajo la hipótesis de que todas las obras se ejecutaron según los plazos contratados.

Como puede verse en la Figura 3 la red predice que las condiciones de orden y limpieza y el estado de los tajos mejorarían en 82 y 53 puntos, respectivamente, bajo estas condiciones.

Por otro lado, bajo la hipótesis de que las condiciones de orden y limpieza eran buenas, Figura 4, la red pronostica que un $28 \%$ más de las obras acabarían en el plazo previsto. Se confirma además que las condiciones del estado de los tajos mejorarían en 16 puntos. 


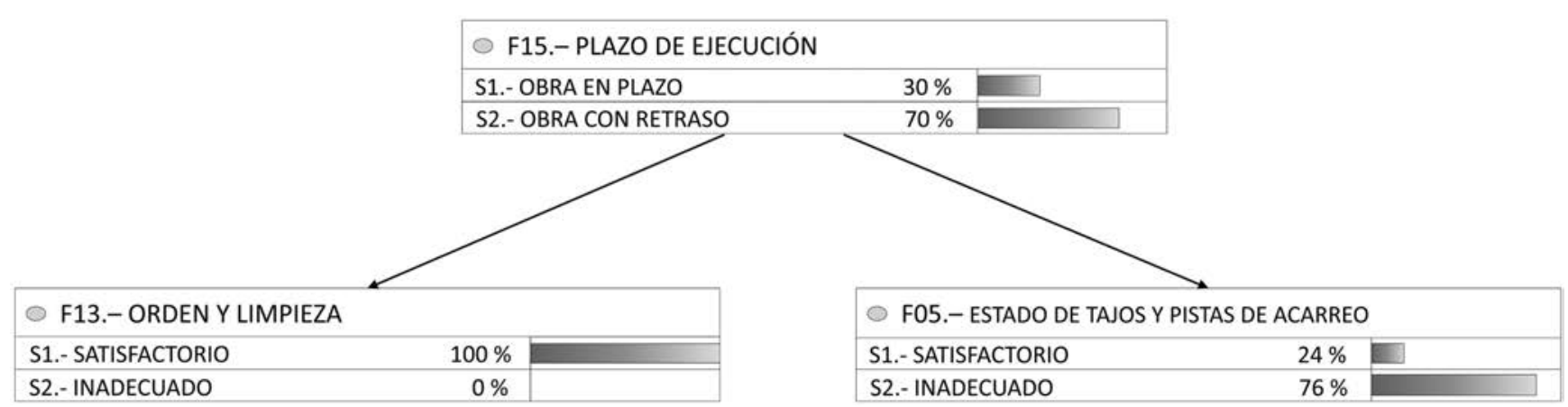

Figura 4. Relación intercausal entre los factores F15, F13 y Fo5 en el movimiento de tierras, bajo la hipótesis de que las condiciones de orden y limpieza eran buenas.

Se muestra, pues, el potencial que tiene la herramienta creada, además del análisis de siniestralidad de las empresas afectadas, la red tiene la capacidad de predicción de posibles perturbaciones en factores que podrían afectar al binomio prevención-producción.

\section{CONCLUSIONES}

En este artículo se ha mostrado un nuevo aporte en el conocimiento de prevención de riesgos laborales en sectores tan exigentes, y de accidentes con consecuencias tan graves, como el de la construcción. En particular, en las actividades de movimiento de tierras.

Dichas actividades, indispensables en el desarrollo de las infraestructuras de cualquier país, necesitan de un nuevo impulso que les permita controlar la siniestralidad manteniendo su nivel de competitividad. El fin último no es otro que el de alcanzar la excelencia preventiva.

Las redes bayesianas se han presentado como importantes herramientas en el análisis de bases de datos y, en particular, para el estudio de casos de accidentes laborales del sector de la construcción. Permiten evaluar el entorno global de las obras de movimiento de tierras o inferir las probabilidades de factores causa y factores efecto en contextos de obra nuevos. También pueden utilizarse para llevar a cabo estudios de sensibilidad, cuantificando el impacto que pequeñas variaciones en alguno de los factores pueden provocar en el resto.

Los resultados obtenidos han servido, por un lado, para identificar y localizar los principales agentes de accidentalidad y, por otro, para identificar los principales factores secundarios que los ocasionan.

En definitiva, la realización de este trabajo ha supuesto la elaboración de una profunda base del conocimiento acerca de una materia, el movimiento de tierras, que todavía tienen un gran margen de mejora en métodos de análisis preventivos.

\section{AGRADECIMIENTOS}

Los autores agradecen la financiación recibida por parte de la Xunta de Galicia y los fondos FEDER a través del proyecto GRC2014/020.

\section{REFERENCIAS}

(1) Swuste, P., Frijters, A., Guldenmund, F. (2012). Is it possible to influence safety in the building sector? A literature review extending from 1980 until the present. Safety Science, 50(5): 1333-1343, doi: http://dx.doi.org/10.1016/j. ssci.2011.12.036.

(2) Feng, Y., Zhang, S., Wu, P. (2015). Factors influencing workplace accident costs of building projects. Safety Science, 72 : 97-104, doi: http://dx.doi.org/10.1016/j.ssci.2014.08.008.

(3) Holte, K. A., Kjestveit, K., Lipscomb, H. J. (2015). Company size and differences in injury prevalence among apprentices in building and construction in Norway. Safety Science, 71: 205-212, doi: http://dx.doi.org/10.1016/j.ssci.2014.01.007.

(4) Lee, H. S., Kim, H., Park, M., Ai Lin Teo, E., Lee, K. P. (2012). Construction risk assessment using site influence factors. Journal of Computing in Civil Engineering, 26(3): 319-330, doi: http://dx.doi.org/10.1061/(ASCE)CP.19435487.0000146.

(5) Park, J., Park, S., Oh, T. (2015). The development of a web-based construction safety management information system to improve risk assessment. Journal of Civil Engineering, 19(3): 528-537, doi: http://dx.doi.org/10.1007/s12205-0140664-2.

(6) Neapolitan, R. E. (2004). Learning Bayesian networks. Prentice Hall.

(7) Rivas, T., Matías, J. M., Taboada, J., Argüelles, A. (2007). Application of Bayesian networks to the evaluation of roofing slate quality. Engineering Geology, 94: 27-37, doi: http://dx.doi.org/10.1016/j.enggeo.2007.06.002.

(8) Martín, J. E., Rivas, T., Matías, J. M., Taboada, J., Argüelles, A. (2009). A Bayesian network analysis of workplace accidents caused by falls from a height. Safety Science, 47: 206-214, doi: http://dx.doi.org/10.1016/j.ssci.2008.03.004.

(9) Li, L., Wang, J., Leung, H., Jiang, C. (2010). Assessment of Catastrophic Risk Using Bayesian Network Constructed from Domain Knowledge and Spatial Data. Risk Analysis: An International Journal, 30(7): 1157-1175, doi: http://dx.doi. $\operatorname{org} / 10.1111 / j .1539-6924.2010 .01429 . x$. 
(10) Rivas, T., Paz, M., Martín, J. E., Matías, J. M., García, J. F., Taboada, J. (2011). Explaining and predicting workplace accidents using data-mining techniques. Reliability Engineering and System Safety, 96: 739-747, doi: http://dx.doi. org/10.1016/j.ress.2011.03.006.

(11) Leu, S. S., Chang, C. M. (2013). Bayesian-network-based safety risk assessment for steel construction projects. Accident Analysis and Prevention, 54: 122-123, doi: http://dx.doi.org/10.1016/j.aap.2013.02.019.

(12) GeNIe \& SMILE (2015). Structural Modeling, Inference, and Learning Engine. Decision Systems Laboratory, University of Pittsburgh, https://www.bayesfusion.com/. 